\title{
On the progress of 3D-printed hydrogels for tissue engineering
}

\author{
Rigoberto C. Advincula (D), Department of Chemical and Biomolecular Engineering and Joint Institute for Advanced Materials, University of Tennessee, Knoxville, \\ TN 37996, USA; Center for Nanophase Materials and Sciences (CNMS), Oak Ridge National Laboratory, Oak Ridge, TN 37830, USA; Department of Macromolecular \\ Science and Engineering, Case Western Reserve University, Cleveland, OH 44106, USA \\ John Ryan C. Dizon, Design, Research, Extension in Additive Manufacturing, Advanced Materials and Advanced Manufacturing (DR3AM) Center/Department \\ of Industrial Engineering, College of Engineering and Architecture, Bataan Peninsula State University, City of Balanga, 2100 Bataan, Philippines \\ Eugene B. Caldona and Robert Andrew Viers, Department of Chemical and Biomolecular Engineering and Joint Institute for Advanced Materials, \\ University of Tennessee, Knoxville, TN 37996, USA \\ Francis Dave C. Siacor, BioProcess Engineering and Research Center and Department of Chemical Engineering, University of San Carlos, 6014 Cebu City, \\ Philippines \\ Reymark D. Maalihan, Chemical and Food Engineering Department and Material Testing and Calibration Center, Batangas State University, 4200 Batangas City, \\ Philippines \\ Alejandro H. Espera Jr., Electronics Engineering Department, School of Engineering and Architecture, Ateneo de Davao University, 8016 Davao City, Philippines; \\ Department of Engineering Education, College of Engineering, Virginia Polytechnic Institute and State University, Blacksburg, VA 24061, USA \\ Address all correspondence to Rigoberto C. Advincula at radvincu@utk.edudec
}

Rigoberto C. Advincula was an editor of this journal during the review and decision stage. For the MRS Communications policy on review and publication of manuscripts authored by editors, please refer to http://www.mrs.org/editor-manuscripts/

(Received 25 April 2021; accepted 12 July 2021; published online: 3 August 2021)

\section{Abstract}

Additive manufacturing or more commonly known as 3D printing, is currently driving innovations and applications in diverse fields such as prototyping, manufacturing, aerospace, education, and medicine. Recent technological and materials research breakthroughs have enabled 3D bioprinting, where biomaterials and cells are used to create scaffolds and functional living tissues (e.g. skin, cartilage, etc.). This prospective focuses on the classification and applications of hydrogels, and design considerations in their production (i.e. physical and biological parameters). The materials for 3D printing of hydrogels, such as biopolymers, synthetic polymers, and nanocomposites, are mainly discussed. More importantly, future perspectives on 3D printing hydrogels including new materials, 4D printing, emerging printing technologies, etc. and their importance in biomedical and bioengineering applications are discussed.

\section{Introduction}

Tissue engineering involves the use of materials engineering and life sciences concepts to regenerate, restore, replace, improve, and maintain tissues damaged by injury, disease, or congenital disabilities. ${ }^{[1]}$ Not only can these procedures be done using basic understanding of the structural and functional relationships of natural and pathologic mammalian tissues, but the development of biosubstitutes can also be achieved. ${ }^{[2,3]}$ Particularly, by harvesting cells from a living organism (or other compatible sources), and seeding the cells onto a tissue scaffold (which becomes the cell-scaffold construct), the construct tends to become a functional construction after maturation. Subsequently, it can be implanted into a human body to help regenerate or repair any damaged tissues. ${ }^{[4]}$ For successful implants and proper tissue scaffold designs, a good understanding of the composition and organization of tissue scaffolds is desirable. Suitable architectural designs and materials capable of mimicking the properties of natural tissues must also be selected. ${ }^{[5]}$ Likewise, proper design towards cell proliferation, cell differentiation, vascularization, and sustainable growth should be implemented. Several groups have recently studied different architectures, materials, growth factors, cell types, and other supporting components to create functional constructs. ${ }^{[6-9]}$ While these attempts need further improvement in mimicking complex tissue structures, challenges of cell differentiation in hierarchical locations or orientation have also been identified. ${ }^{[10]}$

Additive manufacturing (AM), or more popularly known as 3D printing, has tremendously transformed the manufacturing industry since early reports in 1986. AM, which is operationalized from a computer-aided design (CAD), is time- and energyefficient, and generates less waste compared to conventional and formative manufacturing processes. ${ }^{[11-13]} \mathrm{AM}$ is gaining utility in diverse applications such as water filtration and deslination, ${ }^{[14]}$ electronics ${ }^{[15]}$, health ${ }^{[16]}$, prototyping, small production runs, and many others. ${ }^{[11,12,17,18]}$

$3 \mathrm{D}$ printing as employed in tissue engineering ${ }^{[8-10]}$ digitization, has gained medical imaging technology advances in magnetic resonance imaging (MRI), computed tomography (CT), and ultrasound. With personalized medicine, 3D anatomical 
geometry can be extracted from these imaging technologies from which complex tissues can either be replicated, replaced, regenerated, or restored ${ }^{[19,20]}$ - tailor made. For successful reconstruction of human body tissues and organs, artificial transplant supports (i.e. scaffolds) are generally indispensable. ${ }^{[21]} 3 \mathrm{D}$-printed scaffolds provide mechanical support, but also promote cell infiltration and adhesion. ${ }^{[22,23]}$

Hydrogels are currently being used in 3D printing scaffolds due to their ease of chemical design, formulation, and/or functionalization. They can easily replicate the properties of biological tissues with collagen and extracellular matrix (ECM) like properties. ${ }^{[24,25]}$ Hydrogels are 3D polymeric network with hydrophilic chains crosslinked either covalently or physically (via intra- and intermolecular attractions). ${ }^{[26-29]}$ Their hydrophilic structures allow them to hold considerable amounts of water or any aqueous biological fluids up to $10-1000$ times their original weight or volume. ${ }^{[29]}$ Their high water content provides a natural tissue-like environment, providing a hydrated and mechanically stable environment. ${ }^{[30]}$ The presence of crosslinks and/or chain entanglements causes hydrogels to swell readily without dissolution in aqueous environments. The swelling behavior of hydrogels under biological conditions gives them the possibility to diffuse molecules and cells, closer to natural tissues. ${ }^{[31,32]}$ Hydrogels are easily prepared using aqueous chemistries and relatively mild conditions, and are very suitable as scaffold materials due to their versatile structure and composition, which are similar to those of ECM of human tissues. ${ }^{[33,34]}$ In addition, hydrogels exhibit suitable porous structures adaptable to various environmental conditions, can simulate natural ECM, and possess high cell seeding density and homogeneous cell distribution within the scaffold. Hydrogel materials respond remarkably to several stimuli such as water, light, temperature, ions, $\mathrm{pH}$, and biochemical signals. ${ }^{[25,33,34]}$

Yet, there is still a need for further research and development on hydrogel material precursors, crosslinking and hydrogelforming mechanisms, printability, printing parameters, properties (i.e. mechanical, physics, biological, chemical), functionality, and applications - all for optimized 3D printing. This prospective summarizes some of the common challenges and applications of 3D-printed hydrogels for bioimplants and tissue engineering. The printing techniques and materials used are also presented. Future perspectives and identification of challenges and opportunities in 3D printing hydrogels for other biomedical applications are also highlighted.

\section{Brief review of additive manufacturing}

The manufacturing industry has been in a state of transition, shifting to more sustainable, cost-effective, and innovative ways of fabrication. ${ }^{[11,12,15]} \mathrm{AM}$ industries began to rise and have made an impact worldwide even with the current Covid19 pandemic situation. As an advanced manufacturing field, $\mathrm{AM}$ is a tool for a high number of potential applications as it strengthens the research development of materials ${ }^{[35]}$ and rapid product advances. ${ }^{[15,36]}$ By using different 3D printing technologies and customized materials, it has grown to be a more mature, robust, and versatile platform in fabricating geometrically challenging designs, which are otherwise not offered by the traditional manufacturing processes. ${ }^{[32]}$ Here, we briefly introduce the standard AM processes and some of the prominent $\mathrm{AM}$ techniques and technologies used in tissue engineering.

$\mathrm{AM}$, rapid prototyping (RP), 3D printing, and solid freeform fabrication (SFF) are all interchangeably used in the manufacturing space. Figure 1 shows the schematic of these processes and illustrates the general workflow involved in a $3 \mathrm{D}$ printing process. The standard tesselation language (.stl) file format has been used as the standard digital file that takes the information of the surface shape of the three-dimensional model for which it functions as the electronic language between the computeraided design (CAD) software and 3D printing hardware. The model is then sliced into multiple layers, creating a G-file, containing the model's stacked cross-sectional information. This information is finally fed into the specific 3D printing hardware capable of fabricating the model into a tangible product. ${ }^{[14,15]}$ The methods for soft matter or polymer fabrication are generally classified as vat or resin, powder bed, extrusion (ink or filament), and binder jet printing methods. The most popular and widely used polymer 3D printing is called fused deposition modeling (FDM). Various post-processing techniques such as thermal or photo-curing, drying or freezing are employed to ensure the final quality of the printed parts. ${ }^{[11]}$

The biomedical field calls for specific AM techniques including laser printing, extrusion printing, inkjet printing, and 3D bioprinting to fabricate hydrogels for tissue engineering. ${ }^{[9,27]}$ One standard method that falls under the laser-based 3D printing method is stereolithography (SLA), which involves the use of different wavelengths of light (one or two photon) to cure a resin material into high-resolution polymeric parts in a layer-by-layer fashion. ${ }^{[11,12]}$ Digital light projection (DLP) a complementary method can also be utilized. Extrusion or nozzle-based 3D printing, the most popular and promising technique for hydrogels so far, allows materials to be directly extruded through a nozzle tip using either melting (e.g. using

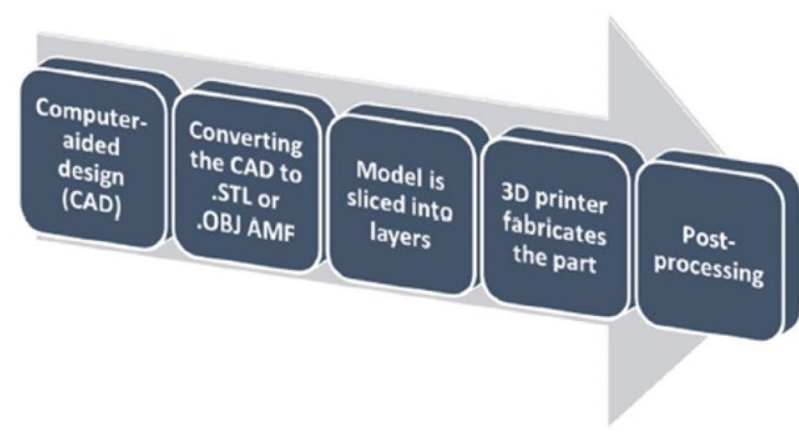

Figure 1. Schematic of the additive manufacturing process flow. ${ }^{[14]}$ 
thermoplastic materials) or non-melting process (e.g. using viscous materials) onto a build plate, where each layer is purposely built upon the previous layer for adherence until the whole part is entirely fabricated. ${ }^{[13,34,37]}$ These methods are known as direct ink writing (DIW) or viscous solution printing (VSP). Inkjet printing is a digital printing process that uses two different processes for 3D printing hydrogels: (1) controlled droplets of liquid material deposited directly onto a substrate that solidifies upon curing; and (2) powder-bed based printing, where the ink, serving as a binder, is deposited from a controlled nozzle to a bed of powder materials bound into multiple layers to create the printed parts. ${ }^{[38]}$ The term $3 \mathrm{D}$ bioprinting employs 3D printing-like fabrication techniques, where biomaterials capable of mimicking natural tissue properties are used to fabricate parts. This technology generally covers a single, combination of, or modified versions of the aforementioned $3 \mathrm{D}$ printing techniques that produce biomedical parts. ${ }^{[4,36]}$ In essence, an appropriate 3D printing method is available in any case for hydrogels and their composites in monomeric, solidstate, and extrudable gel forms, all of which can fit towards the chemical and physical process of crosslinking during printing.

\section{Hydrogels for tissue engineering applications Classification and applications of hydrogels}

Hydrogels are arguably the best candidate materials for tissue engineering applications due to their previously discussed characteristics. Prior to briefly describing their utility in an array of tissue engineering applications, it is worthy to note the different classifications of hydrogels. This classification is essential as it affects the conditions for method preparation and feasibility of exploiting hydrogels, based on their intrinsic physical and chemical characteristics.

Hydrogels are classified based on their sources (i.e. natural, synthetic, and hybrid). Natural hydrogels can be derived from proteins (e.g. collagen, elastin, fibrin, gelatin, silk fibroin, etc.), polysaccharides (e.g. glycosaminogylcans, alginate, chitosan, etc.), and decellularized tissues. Synthetic hydrogels exhibit more versatile and easily-controlled physical and chemical properties compared to natural alternatives ${ }^{[28]}$ by virtue of molecular and macromolecular design. Examples of synthetic hydrogels are polylactic acid (PLA), polyglycolic acid (PGLA), polyethylene oxide (PEO), polyethylene glycol (PEG), polycaprolactone (PCL), polyacrylates, polyvinyl alcohol (PVA), polyvinylpyrrolidone (PVP), polyimide (PI), and all their respective derivatives. Hybrid hydrogels are a combination of natural and synthetic materials, composed of composite structures with desirable synthetic and natural characteristics. ${ }^{[39]}$ Hydrogels, based on their structural integrity, can be durable or biodegradable. Durable hydrogels are usually synthetic and are mechanically stronger compared to naturally derived hydrogels, while biodegradable hydrogels are natural polymers, generally nontoxic, and demonstrate less adverse effects compared to synthetic alternatives. ${ }^{[40]}$ Lastly, hydrogels can be further categorized based on their environmental stimuli-responsiveness. Popularly known as smart hydrogels, stimuli-responsive hydrogels are capable of reversibly changing their shape and volume in response to changes in $\mathrm{pH}$, temperature, light, and electric and magnetic fields, thereby altering their physicochemical characteristics. ${ }^{[41]}$ Many recent studies have geared towards the utility of hydrogels for various tissue engineering applications. Different fabrication approaches including layer-by-layer (LbL) assembly, ${ }^{[42]}$ microfluidic fabrication, ${ }^{[43]}$ electrospinning, ${ }^{[44]}$ self-assembly, ${ }^{[45]}$ and $3 \mathrm{D}$ printing ${ }^{[46]}$ have been widely employed to optimize process parameters, which can result in a desirable hydrogel functionality. Specifically, for hydrogel 3D printing, 3D plotting, ${ }^{[47]}$ direct ink writing, ${ }^{[9]}$ and robocasting ${ }^{[48]}$ are used for fabrication of scaffolds with complex architectures in the wet state. A summary of different polymeric hydrogel materials, cell types being used, target tissue applications, and the corresponding fabrication methods is presented in Table I. In a nutshell, hydrogels for tissue engineering applications are practically useful as scaffolds capable of mimicking native extracellular matrices, supporting the growth of new cells, and promoting the repair of damaged tissues.

Table I. Recent polymer-based hydrogels for tissue engineering applications.

\begin{tabular}{|c|c|c|c|c|}
\hline Type of polymer & Cell type used & $\begin{array}{l}\text { Target tis- } \\
\text { sue applica- } \\
\text { tion }\end{array}$ & Fabrication Technique & Reference \\
\hline $\begin{array}{l}\text { Alginate/bacterial cellulose nanocrystals-chi- } \\
\text { tosan-gelatin }\end{array}$ & MC3T3-E1 cells & Bone & $\mathrm{LbL}$ & Yan et al. ${ }^{[42]}$ \\
\hline Gelatin & Human umbilical endothelial cells & Vascular & Microfluidics & He et al. ${ }^{[43]}$ \\
\hline $\begin{array}{l}\text { Poly(ethylene glycol)-poly( } N \text {-isopropylacryla- } \\
\text { mide) (PEG-PNIPAAm)-poly( } \varepsilon \text {-caprolactone) }\end{array}$ & Human mesenchymal stem cells & Cartilage & Electrospinning & Brunelle et al. ${ }^{[44]}$ \\
\hline Peptide & Fibroblasts, dental pulp stem cells & Dental pulp & Self assembly & Nguyen et al. ${ }^{[45]}$ \\
\hline Chitosan/PVA & Human mesenchymal stem cells & Bone & 3D printing (extrusion) & Ergul et al. ${ }^{[46]}$ \\
\hline
\end{tabular}




\section{Design criteria for hydrogels in tissue engineering}

Hydrogels are expected to meet several design criteria for them to properly function, stimulate new tissue formation, and induce minimal to no immune reaction from the recipient. Satisfactory design and materials selection for hydrogels depend mainly on their physical parameters (i.e. mechanical properties, biodegradability/biosorbability, porosity, and swelling behavior) and biological performance (i.e. biocompatibility, cell adhesion, vascularization, and bioactivity). These factors are defined by their intended application and environmental exposure. For instance, hydrogels intended for artificial skin production must be different from those employed for artificial bones. Thus, the utility of a particular hydrogel structure and composition varies over a range of tissue engineering applications.

\section{Physical parameters}

a. Mechanical properties In tissue engineering applications, it is imperative to match the hydrogel mechanical properties with those of the tissues in the implantation site. Such properties can be tailored to meet specific end-use requirements. For instance, the mechanical strength of hydrogels may be increased by inclusion of crosslinking agents, ${ }^{[49]}$ block copolymerization, ${ }^{[50]}$ formation of interpenetrating networks (IPNs) ${ }^{[51]}$ or semi-interpenetrating networks (SIPNs), and addition of nanofillers such as graphene, nanosilica, carbon nanotubes, and their derivatives. ${ }^{[52,53]}$ The presence of nanofillers, however, generally produces a lowstrength hydrogel with low elongation percentage at increasing nanofiller loading, leading to a brittle structure. Thus, an inevitable trade-off between mechanical strength and flexibility is expected for hydrogels to properly function as tissue-engineered scaffolds. Gel elasticity is also important as it provides crosslinked chain flexibility and facilitates the movement or diffusion of bioactive agents. ${ }^{[54]}$

b. Biodegradability/biosorbability Ideally, hydrogels should exhibit bioresorbability and tunable degradation and resorption rates upon formation of functional tissues as a result of new cell replacement. Maintenance of both the cellular proliferation and desired distribution is one of the basic requirements of hydrogels during their anticipated lifetime, ${ }^{[40,41,44]}$ which lasts upon their complete biodegradation. Hence, biodegradation rates are crucial in designing effective and useful hydrogels for tissue engineering applications. Rapid hydrogel degradation may lead to higher resorption rates prior to production of sufficient tissues, whereas slow degradation may impede tissue formation.

c. Porosity Hydrogel porosity shows the presence of void cavities inside its bulk structure. A substantial amount of scaffold porosity or hierarchical transport properties is important for efficient nutrient and metabolic waste transport and optimal cell migration. Hydrogels should contain enough interlinked porous network to facilitate proper cell growth and flow, and introduce increased surface area tantamount to the desired scaffold volume. Such extreme, interconnected porosity can provide benefits to cell ingrowth and uniformity, especially in the absence of a functional vascular system. ${ }^{[27,28]}$

d. Swelling Swelling is intrinsically characterized by the hydrogel ability to enlarge as a result of solvent permeation through the void spaces between the polymeric chain networks. Swelling is commonly associated with the material's mechanical properties, which, in turn, show correlation with the hydrogel composition and crosslinking density. Additionally, swelling plays a crucial function in materials diffusion and transport within and through the hydrogel, both of which are essential for encapsulated cell stability and molecule release for drug delivery. ${ }^{[31,39]}$ Both the swelling capacity and rate are critical as they can influence the usability of hydrogel materials. It is also important to reckon the swelling rates for specific in vivo applications as they can provide a glimpse of defect filling rates throughout a particular surgical procedure. ${ }^{[27]}$

\section{Biological performance}

a. Biocompatibility Biocompatibility is of primary interest to researchers seeking to develop hydrogels with end uses in biological systems. Biocompatibility typically refers to the capability of a material to be integrated into a biological system without harming or being rejected by the said system. ${ }^{[55]}$ As a biomaterial, hydrogels should be designed and produced with minimal or no immune reactions towards the living tissues. Biocompatibility is characterized by simultaneous hydrogel matrix degradation and formation of new tissues. ${ }^{[41]}$ The matrix should be safe and noncytotoxic physiologically. Various types of synthetic polymers, including polyesters and acrylates, have been reported to display biocompatibility with the human body. ${ }^{[54]}$ Poly(amino acids), hyaluronic acid, and other bioderived materials have also been used to fabricate biocompatible hydrogels. ${ }^{[56]}$ It is noteworthy that these hydrogel precursor polymeric materials, in their pristine form, should not only be biocompatible, but also fully polymerizable prior to hydrogel use and start of any degradation.

b. Cell adhesion Hydrogel scaffolds are expected to display adhesion property for cell binding. Note that many synthetic polymers possess low cell bioadhesion. Poly(2-hydroxyethyl methacrylate) (PHEMA), PEG, and PVA are among the most commonly employed synthetic polymers in tissue engineering that show weak cell binding. ${ }^{[57]}$ Derivatization, via composite scaffold formation or functionalization with bioactive 
motifs and imprinting (e.g. RGD peptides), is therefore necessary to facilitate cell adhesion.

c. Vascularization Implanted tissues possessing higher survivability are vascularized where capillary network responsible for nutrients transport to the cells is grown in the tissue. Proper scaffold design, angiogenic factors, and pre-vascularization (both in vivo and in vitro) are commonly employed strategies for enhanced vascularization. ${ }^{[58]}$ Among these approaches, proper hydrogel scaffold designs with adequate interconnectivity, branching and precise pore size have been found to influence the post-implantation vascularization rate. ${ }^{[59,60]}$

d. Bioactivity Bioactivity refers to the ability/propensity of an implant material to trigger/facilitate a biological response within a living system upon introduction (e.g. tissue bonding in the human body) ${ }^{[55]}$ Desirable features of a typical bioactive scaffold include tissue interactivity and binding ability, excellent osteoconductivity and osteoinductivity, and cell differentiation, attachment, and ingrowth stimulated by incorporating growth factors and biological cues. ${ }^{[66]}$ As a result of their unique structure, hydrogels are also capable of controlling and sustaining the release of bioactive factors, promoting continuous vascular network and bone matrix formation. Structural modification and tuning the concentrations of polymers and crosslinkers can lead to controlled hydrogel degradation and molecular diffusion rates. ${ }^{[61]}$

\section{Challenges in the production of hydrogel structures}

Various tissue-engineered devices that underwent successful clinical research and trials are still confronted with several substantial challenges and limitations. These issues arise from the utility of artificial hydrogel scaffolds and their corresponding fabrication methods to imitate natural ECMs ${ }^{[41]}$ which assist in the propagation, differentiation, and biosynthesis of cells. ${ }^{[62]}$ For hydrogel structures manufactured through 3D-printing, enhanced printers to date are able to achieve acceptable printing resolution $(\sim 0.3 \mathrm{~mm})$. With this resolution, these printers can be applied in bone tissue engineering, in which approximately $0.2-0.35 \mathrm{~mm}$ scaffold pore size is required ${ }^{[1]}$ However, these printers, such as inkjet thermal 3D bioprinters, impart localized heating from around $200-300^{\circ} \mathrm{C}$ corresponding to a temperature rise of $4-10^{\circ} \mathrm{C}$. But even then, heating did not impart significant effect on the stability of hydrogel scaffolds. ${ }^{[4]}$

One of the challenges of these engineered devices is the inferior performance of cell infiltration, resulting in uneven cell seeding. This finding is largely attributed to the pore size and structure, and the absence of suitable controls in spatial and temporal aspects concerning cell migration and proliferation. ${ }^{[63 \text {, }}$

${ }^{64]} \mathrm{A}$ phenomenon known as spatial inhomogeneity is exhibited when hydrogels display inhomogeneous crosslink density distribution, resulting in the reduction of hydrogel strength. ${ }^{[65,66]}$
There are also difficulties associated with the integration of hydrogel systems in the human body since it needs to behave consistently and rhythmically with the different pathological and physiological occurrences, as well as the physical and biochemical properties of several organs. ${ }^{[33,67]}$ Although hydrogels are now considered smart materials and their manufacturing techniques are more advanced, their vascularization needs to be addressed such that their behavior should pattern the complex structure of natural tissues. Consequently, this action would disrupt the barrier in the production of intricate organs. ${ }^{[64]}$

The qualities of biomaterials, specifically its mechanical properties, have a small but significant impact when utilized as a scaffolding material. This means then that when constructing appropriate scaffold constructs incorporating biomaterials, the goal should be to convert the mechanical characteristics of the target tissue into the mechanical characteristics of the manufactured construct. With this, it was then found that mechanical strengths equal to $10-1500 \mathrm{MPa}$ is required for hard tissue regeneration while soft tissue regeneration necessitates strength within $0.4-350 \mathrm{MPa} \cdot{ }^{[65]}$ In the case of hydrogel systems, its mechanical properties are found to be poor at both macro- and microscopic levels. Hence, their resistance to mechanical stress is relatively low because their network is assembled through the asymmetrical arrangement of crosslinkers. ${ }^{[68]}$ As a consequence, their applications are still limited to soft and non-load bearing tissues. To further enhance the mechanical properties of their structures, additives such as nanoparticles and polymer matrices are embedded in hydrogels during fabrication. This structural modification may potentially result in ECMs having structure and function similar to their native forms. Besides, hydrogel systems tend to be fragile and delicate in terms of handling and loading. ${ }^{[65,69]}$

Furthermore, synthetic hydrogels pose biocompatibility and biodegradability issues. The crosslinked structures of hydrogels formed through chain-growth addition polymerization can produce high molecular weight and viscous materials. As viscosity is further increased, monomers are slowly diffused, thus hindering their complete consumption and resulting in residual monomers in the structure, which are often contaminated. Accordingly, sterilization is negatively impacted and may further become complicated with the presence of residual water molecules. Finally, the degradation rates of hydrogels when used either as scaffolds or in regenerative medicine are still inconsistent with those of their native forms, challenging the biodegradability of hydrogels. ${ }^{[31,64,70]}$

\section{Opportunities in the production of hydrogel structures}

The production of hydrogel structures via 3D printing presents several advantages. It is able to print porous and complex features which allows interconnection of micropore structure. Exploiting this fabrication method in the area of tissue-engineering affords improvement in the transport of nutrients, removal of waste and oxygen exchange within cells, cell migration and cell proliferation. For oxygen exchange to 
achieve efficiency, pore sizes should be optimal at $\sim 100 \mu \mathrm{m}$ through a fine nozzle having $100 \mu \mathrm{m} .{ }^{[8]}$ Hence, advancement of 3D-printing technologies should be anticipated to increase pore resolution while maintaining geometry, mechanical properties and stability of scaffolds. This would mean targeting anatomical features of tissue architectures on the scale of $>100 \mu \mathrm{m}$. It has been demonstrated that scaffolds having a maximum of $200 \mu \mathrm{m}$ pore size efficiently transport oxygen to cells. ${ }^{[21]}$ Moreover, microextrusion-based 3D printers are compatible with a wide array of biomaterials with viscosities from 30 to $>6 \times 10^{7} \mathrm{mPa} / \mathrm{s}$. This would imply flexibility of $3 \mathrm{D}$-printing methods, which can accommodate materials with higher viscosity. These materials provide structural support for tissue-engineered devices. ${ }^{[36]}$

To circumvent the limitations in mechanical properties, novel types of functional nanomaterials are considered in developing nanocomposite hydrogels with enhanced polymernanomaterial interfacial interactions, leading to improved hydrogel structure and thermo-mechanical stability. There are also concerns towards the uneven dispersion of nanomaterials upon addition to the hydrogel matrix, but such issues can be addressed by proper surface matrix modification. ${ }^{[68]}$

The biocompatibility issues of hydrogel structures can be resolved via the integration of conductive polymers. Wu et al. ${ }^{[71]}$ showed that electroconductivity can potentially lead towards substantial improvement in neuron elongation of neural tissues. The incorporation of electroconductive materials can modify, not only the physical properties, but also electrical and dielectric properties of the engineered neural tissue. In another study, ${ }^{[72]}$ the inclusion of electroconductive materials in engineered cardiac tissues significantly improved cell elongation and alignment, and intercellular coupling. These findings are supported by Navaei et al. ${ }^{[73]}$ where beating frequency and excitation threshold were also found to have improved. Moreover, the biodegradation of hydrogels should be appropriately assessed using in vivo tests due to difficulty in achieving natural human body environment during in vitro studies. This evaluation would enable other degradation products to be identified, making it easier to study their distribution in the hydrogel structures. ${ }^{[74]}$ Novel strategies in the production of exceedingly biomimetic and structurally-designed nanocomposite hydrogels are also being explored.

\section{D-Printed hydrogels for tissue engineering}

Tissue engineering has emerged as an area of particular interest and promise, particularly for hydrogels largely because of their capability to facilitate cell adhesion, proliferation, and differentiation. Hydrogels serve as an ideal medium for the efficient exchange of nutrients and waste with surrounding tissues, in which they can also function as scaffolds due to their biocompatibility and sufficient mechanical properties. ${ }^{[75]}$ The combination of these properties justifies continued research of polymer hydrogels for tissue engineering

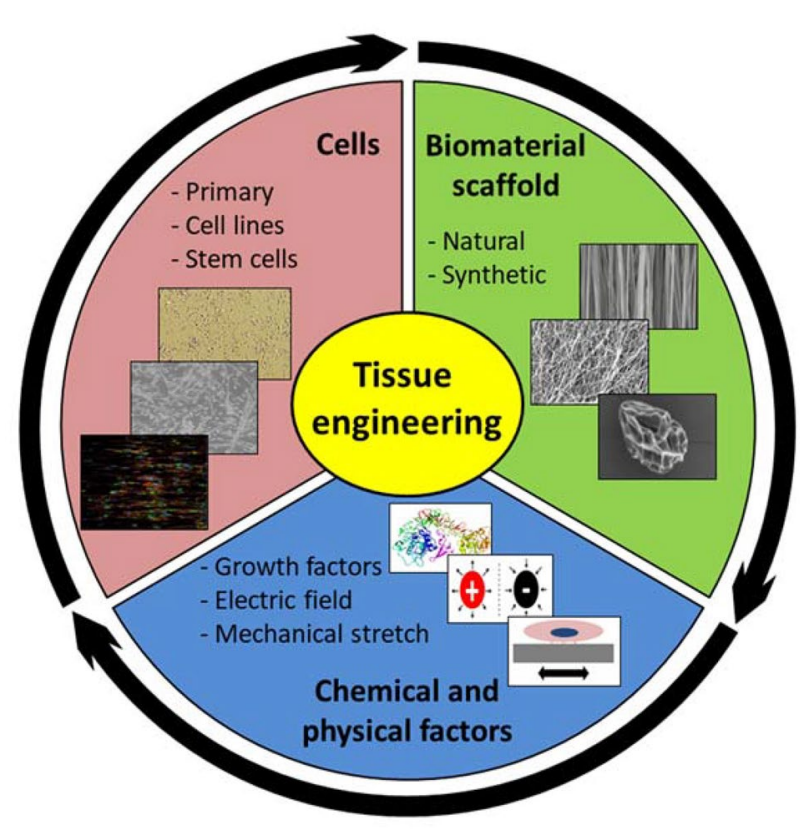

Figure 2. The fundamental stages of tissue engineering: the introduction, culturing, and development of cells on a bioscaffold, providing them makeup with physiochemical properties relevant to tissue regeneration. ${ }^{[76]}$ Licensed under Creative Commons: https://creativecommons.org/licenses/by/4.0/).

applications. Figure 2 illustrates the different phases and considerations required to successfully develop materials with applications in tissue engineering. ${ }^{[76]}$ Biopolymers, synthetic pristine polymers, and nanocomposite materials have been investigated as starting materials for hydrogel fabrication. ${ }^{[9]}$ Each of these precursors display several advantages and disadvantages when used to create hydrogels for tissue engineering applications. For instance, biopolymers, are often biocompatible and bioactive, but typically exhibit inherent structural features, which may be difficult to replicate in a laboratory setting. ${ }^{[35]}$ By contrast, using synthetic polymers for hydrogel formation allows for targeted selection and modification of properties including molecular weight and morphology. Similarly, nanocomposites and polymer blends can be tailored to improve the properties necessary for a given hydrogel application.

Given the material and technical advantages and possibilities, 3D printing of hydrogels enables a wide range of customized, patient-specific biomedical applications. Hydrogel materials often require relatively mild processing conditions and thus, not all AM modes are compatible with them. As previously discussed, SLA laser-based printing techniques employ photopolymerization mechanisms in curing photoactive hydrogels to form solids with the desired geometry. ${ }^{[9]}$ Extrusion-based printing modes are functionally analogous to industrial extruding processes, where the polymeric material is processed at temperatures necessary to induce a viscous flow. Subsequently, the material is deposited layer-by-layer to create a predefined 
structure. Likewise, printer-based printing techniques, such as inkjet printing, are also used to print polymer hydrogels. Inkjet printing is a contactless reprographic technique, which works by obtaining digital information from a computer and subsequently replicating the information onto a substrate via ink droplets. ${ }^{[9]}$ This section introduces various materials and processes used to $3 \mathrm{D}$ print hydrogels for applications in tissue engineering.

\section{D printing natural biopolymers}

Natural biopolymers regularly exhibit properties that are difficult, often impossible, to replicate by synthetic approaches and are frequently more amenable to biological environments when compared to synthetic polymers. ${ }^{[35]}$ Collagen, gelatin, and alginate are among the most studied naturally occurring hydrogel polymers for biomedical implications. The challenge is to adapt or modify these materials without compromising their native or hierarchical structures (secondary, tertiary, and quaternary).
Collagen constitutes a significant portion of the extracellular matrix (ECM), allowing for the extraction of printable collagen in large quantities. Collagen also demonstrates exceptional biocompatibility and biodegradability. ${ }^{[35,77]}$ These properties have encouraged researchers to advance the ability of collagen to be directly $3 \mathrm{D}$ printed or modify them with other polymers as blends or copolymers.

Martin et al. ${ }^{[78]}$ demonstrated the potential for modified PLA-collagen (PLA-Col) scaffolds to facilitate cell adhesion, proliferation, and osteogenic activity, while limiting unwanted bacterial formation over the surface of the scaffold. The PLA modified collagen scaffolds were $3 \mathrm{D}$ printed and subsequently coated with bioactive collagen, minocycline (MH), and citratehydroxyapatite nanoparticles (cHA). $\mathrm{MH}$ demonstrates antibacterial properties, while $\mathrm{cHA}$ improves the adhesion and proliferation of stem cells derived from human bone marrow on the scaffold surface. Various characterization techniques indicated that the PLA-Col-MH-cHA scaffolds exhibited physical and (a)

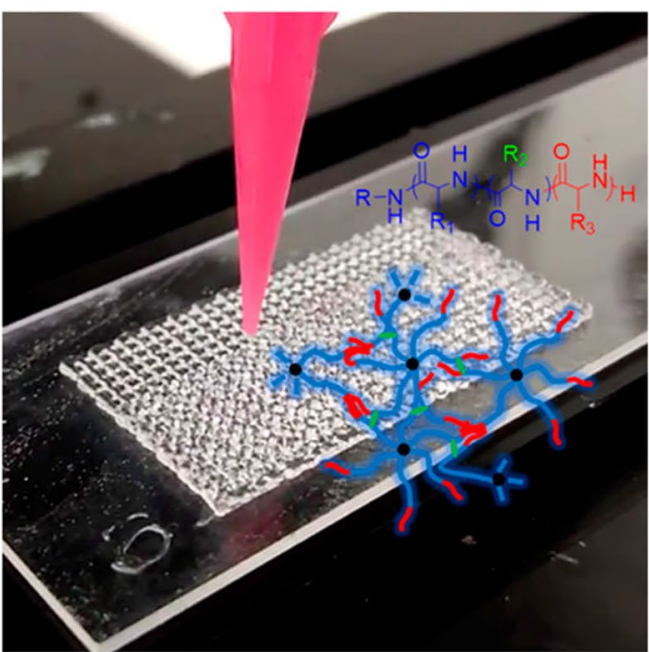

(b)

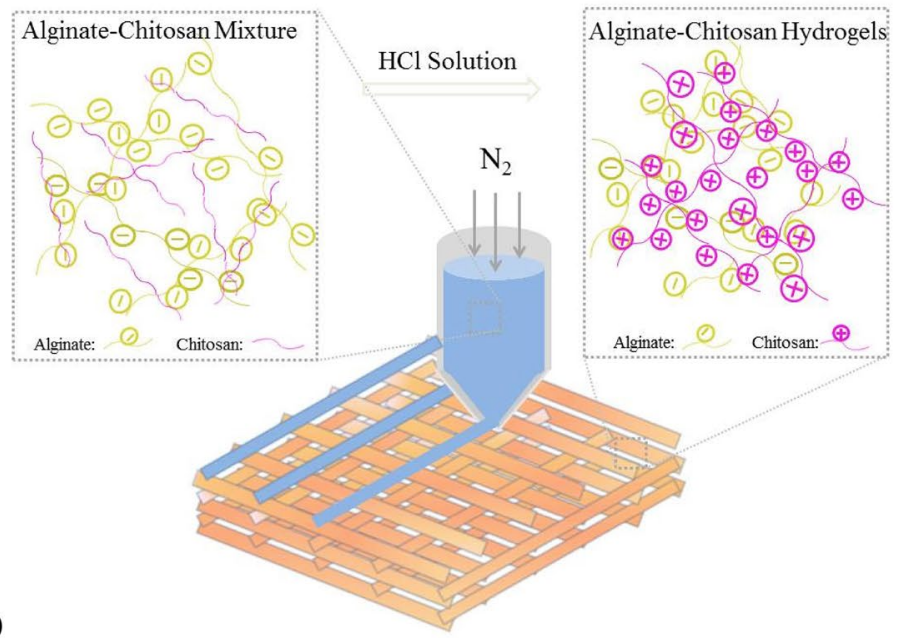

3D Printed PEGDA Hydrogel Platform

(c)

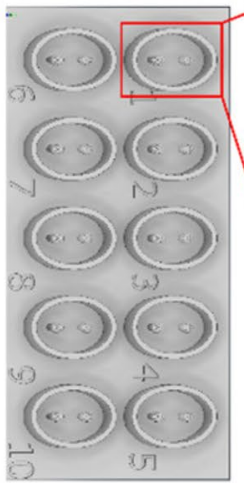

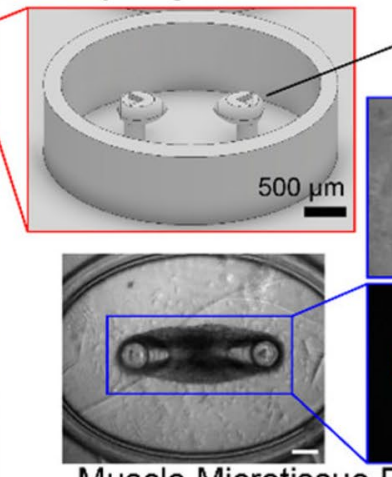

Muscle Microtissue From Fibrin Hydrogel
Stiffness-by-Design Low Tissue Stress Integrated Markers

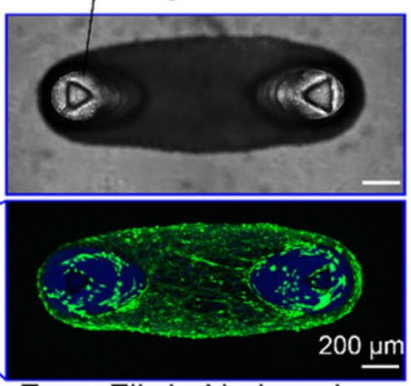

00 (d)

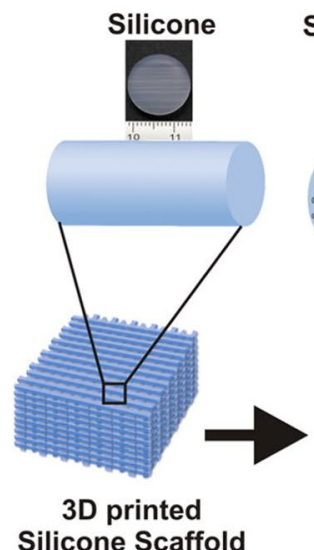

Silicone-hydrogel

IPN
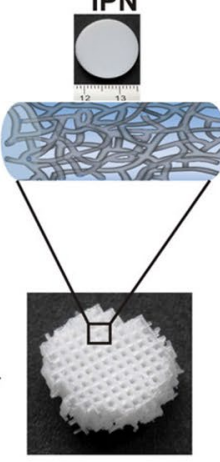

3D IPN Scaffold

Figure 3. (a) Extrusion-based 3D printing of star-shaped dendrimer-based hydrogel. ${ }^{[79]}$. Reprinted (adapted) with permission from Biomacromolecules 2018, 19, 7, 2691-2699. Copyright (2018) American Chemical Society; (b) Extrusion-based 3D printing of alginate/chitosan hydrogel ${ }^{[81]}$; (c) SLA 3D printed PEGDA hydrogel structures. ${ }^{[83]}$ Reprinted (adapted) with permission from Biomacromolecules 2020, 21, 2, 356-365. Copyright (2019) American Chemical Society; (d) 3D printed silicone-based hydrogel IPN scaffold. ${ }^{[84]}$ Reprinted (adapted) with permission from Biomacromolecules 2016, 17, 4, 1321-1329. Copyright (2016) American Chemical Society. 
biological properties more closely resembling that of natural bone architectures.

Murphy et al. ${ }^{[79]} 3 \mathrm{D}$ printed star-shaped dendrimers composed of polypeptide copolymer blocks using extrusion-based printing techniques [Fig. 3(a)]. The process was coupled with post-print UV treatment to form mechanically stable hydrogels with controlled microstructures. Using L-glutamate and L-valine functionality resulted in degradable hydrogels incapable of inhibiting the functionality of the Balb/3T3 cell line. The degradation was exploited by loading the hydrogel with the small molecule drug doxorubicin hydrochloride to demonstrate the potential for controlled drug release.

Wang et al. ${ }^{[00]}$ used digital light projected (DLP) 3D printing techniques to construct stable hydrogel structures from thiolated heparin mixed with glycidyl methacrylated hyaluronic acid and various growth factors. Various geometries were constructed in a layer-by-layer fashion, where every other layer contained growth factors to study the spatial effect on controlled growth factor release. Results demonstrated that the presence of heparin coupled with various geometries altered the kinetics of growth factor release in hydrogel media.

Liu et al. ${ }^{[81]}$ showed that alginate solutions modified with chitosan powder exhibit viscosities necessary for extrusionbased 3D printing[(Fig. 3(b)]. The mechanical strength was improved by further modification with $\mathrm{Ca}^{2+}$ for ionic crosslinking, which resulted in a compression strength of $1.4 \mathrm{MPa}$ at $90 \%$ strain rate. Biocompatibility was confirmed upon introduction of pluripotent human adipose-derived stem cells. On the other hand, fluorescence microscopy confirmed the proliferation and adhesion of the stem cells on the hydrogel surface.

\section{Synthetic hydrogel polymers}

Synthetic polymers have also gained ground as viable printable materials for the construction of de novo hydrogels in tissue engineering. While many synthetic polymers do not demonstrate the same level of biocompatibility and bioactivity as natural biopolymers, there are advantages of using synthetic polymers. The utility of synthetic polymers in fabricating 3D printed polymer hydrogels affords choices that are not available when using natural biopolymers. For instance, a wide variety of monomers and crosslinkers can be selected based on specific target properties including molecular weight, degree of functionalization, and surface morphology. ${ }^{[9]}$ By adjusting these parameters, a given synthetic polymer can be optimized for a specific application. Examples of synthetic polymers that have been used to successfully 3D print hydrogels include polyacrylamide, PVA, and PEG derivatives. ${ }^{[9]}$

PEG has emerged as a material of choice for hydrogel formation in tissue engineering applications because of its exceptional biocompatibility. PEG is hydrophilic and hydrolytically stable, making it suitable for use as biological matrix. It is also resilient against cell adhesion and protein adsorption, permitting more targeted control of tissue growth. ${ }^{[9,82]}$ The molecular weight of PEG can be tightly controlled, allowing physical properties to be adjusted appropriately for a given application.
PEG can be safely dispelled by the body due to limited digestion of the polymer chain. ${ }^{[9]}$ PEG has also been shown to be compatible with other polymerizable species, allowing the formation of copolymers. This compatibility introduces a control dimension in modifying the properties of PEG-derived polymers. ${ }^{[82]}$ Regions, or blocks, of a specific polymer backbone can be added to improve mechanical properties like toughness and impact strength. Careful selection of PEG copolymers is necessary to ensure the preservation of biocompatibility. Adjacent block chains should be degradable for PEG blocks to be released. $^{[82]}$

Christensen et al. ${ }^{[83]}$ fabricated high resolution poly(ethylene glycol) diacrylate (PEGDA) hydrogel structures using SLA 3D printing technique [Fig. 3(c)]. Micro-cantilever structures imbedded in a cell laden hydrogel matrix were used to facilitate tissue growth with tunable features being afforded by varying PEGDA concentration and cantilever diameter. This tunability enables tissue strips to form without being damaged due to excessive stress about the cantilevers. Biocompatibility and controlled cellular proliferation were confirmed using fluorescent microscopy.

Tessmar and Göpferich ${ }^{[82]}$ reviewed a variety of PEG-copolymer moieties for applications in tissue engineering. Highly hydrated PEG-based polymers and crosslinking functionality were mostly employed with the aim of producing biomimetic properties necessary for cell proliferation. Using specific short peptide chains as crosslinkers allowed for selective cleavage of the crosslinked groups upon introduction of certain proteinases. Crosslinked peptide sequences can also be selected to have regions recognized by receptor substrates resulting in increased cellular adhesion. The targeted cellular adhesion can result in guided migration throughout the scaffolded network.

Mohanty et al. ${ }^{[84]}$ employed an indirect 3D printing approach (i.e. casting and supercritical fluid technique) in producing IPNs from silicone and other Food and Drug Administration (FDA) approved polymer materials. Silicone-poly(2hydroxyethyl methacrylate)-co-poly(ethylene glycol) methyl ether acrylate, the resulting 3D printed scaffold [Fig. 3(d)], allowed for viable stem cell growth and metabolic activity. The porous microstructures of the hydrogels were also used to study the potential for controlled drug release, and it was found that doxycycline-loaded IPNs triggered gene expression of HeLa-Tet-On cells.

Janarthanan et al. ${ }^{[85]}$ showed that hyaluronic acid (HA) functionalized with 3-amino-1,2-propane diol can take on aldehyde functionality when reacted with sodium periodate, and subsequently crosslink with carboxymethylcellulose (CMC) functionalized with carbohydrazide $(\mathrm{CH})$ through the formation of an acyl-hydrazone bond. The resulting hydrogel, fabricated through an extrusion-based 3D printing technique or DIW, exhibited high fidelity capable of being processed into lattice, cube, and tube structures. In vitro studies demonstrated self-healing properties, drug delivery capability, and noncytotoxicity. In vivo studies in mice were also performed and results showed that injecting mice with the HA-CMC hydrogels 
subcutaneously caused increased angiogenesis after 4 weeks, compared to the saline control.

\section{Nanocomposite materials}

Hydrogels derived solely from naturally occurring or synthetic polymers often lack the desired mechanical strength required for certain applications in tissue engineering. The utility of nanocomposite materials improves the structural integrity of the hydrogel by integrating nanoparticles, usually of organic or inorganic origin, into the hydrogel matrix. ${ }^{[9]}$ This added mechanical strength substantially expands the biomedical applications of hydrogels to potentially treat conditions like bone defects, while also introducing the possibility for applications in electronics and soft robotics. ${ }^{[86]}$

Wet hydrogel structures consisting of $98 \mathrm{wt} \%$ water and $2 \mathrm{wt} \%$ cellulose nanofibrils (CNFs) were successfully 3D printed using an extrusion-based printing technique. ${ }^{[87]}$ The resulting printed structures were subject to various postprint drying methodologies to investigate the effects on the physical properties and dimensional retainment. Results on mechanical testing indicated that air-dried structures in the presence of a surfactant gave a significantly higher tensile strength and mean stiffness compared to that of the freezedried parts. Drying structures in the presence of a surfactant also resulted in improved dimensional retainment. CNFs loaded with carbon nanotubes (CNTs) were also printed by the same technique to produce structures with significantly higher conductive properties compared to that of the pristine CNF structures. The ability to print wet hydrogels and introduce conductive functionality affords many potential applications for CNF composite materials in the biomedical and tissue engineering space.

Iron oxide magnetic nanoparticles (MNPs) coated with PEG-nitro dopamine were employed at low concentrations to mechanically reinforce collagen-based hydrogels. ${ }^{[88]}$ The resulting hydrogels could be tuned to have a mechanical stiffness between 0.2 and $200 \mathrm{kPa}$ by varying the concentration and particle size of the MNPs. The improved mechanical properties at low concentrations are attributed to the functionalized MNPs serving as crosslinking epicenters within the hydrogel network. The resulting structures also demonstrated biocompatibility when loaded with human bone marrow stem cells. No changes in metabolic activity within the cells were observed and proliferation occurred within $72 \mathrm{~h}$. Cells were observed to respond to external stresses on the hydrogel matrix. Table II summarizes the aforementioned material precursors, along with the cell types and target applications, in 3D printing tissue-engineered hydrogels.

\section{Hydrogel-based bioinks}

Applications of 3d-printed hydrogel-based bioinks include the fabrication of tissues such as skin, musculoskeletal tissue, cardiac tissue, heart valve, and neuronal tissue. Hydrogel polymers used include collagen, agarose, alginate, hydroxyapatite, polycaprolactone, gelatin and polyacrylamide. Conventional approach to tissue engineering has many limitations which may be addressed by $3 \mathrm{~d}$ printing. The following techniques have been used in the $3 \mathrm{~d}$ printing of hydrogel-based bioinks: Inkjet bioprinting, thermal inkjet bioprinting, piezoelectric inkjet bioprinting, microextrusion bioprinting, and laser-assisted bioprinting ${ }^{[89]}$

\section{Challenges and opportunities}

Researchers hope to infuse 3D printed scaffolds with the appropriate bioactive components so that in the future, patients in need of transplants no longer wait for organ donors. If this is achieved, customizable 3D printed parts can be manufactured for patients in a much shorter, more predictable time frame. Figure 4a illustrates a 3D printed model human heart using collagen, a promising candidate for use in hydrogel scaffold formation in tissue engineering applications. ${ }^{[90]}$ While the technology and understanding needed to turn a replica like this into a functioning human heart is not yet available, the intention of the research is apparent and would have massive implications for addressing issues of heart defects and failures. Since heart failure is considered a major cause of death worldwide, a functioning technology of this kind could significantly extend the average human lifespan.

Table II. Materials used in 3D printing hydrogels for tissue engineering applications.

\begin{tabular}{|c|c|c|c|c|}
\hline Material & Cell type & Target application & $\begin{array}{l}\text { 3D-print- } \\
\text { ing tech- } \\
\text { nique }\end{array}$ & Reference \\
\hline PLA-collagen & Human mesenchymal stem cells & Osteogenesis & FDM & Martin et al. ${ }^{[78]}$ \\
\hline Block copolypeptides & Balb/3T3 & Stroma generation & Extrusion & Murphy et al. ${ }^{[79]}$ \\
\hline Thiolated heparin & - & Controlled growth factor release & DLP & Wang et al. ${ }^{[80]}$ \\
\hline Ionically crosslinked alignate & Human adipose-derived stem cells & Adipose/bone tissue generation & Extrusion & Liu et al. ${ }^{[81]}$ \\
\hline PEGDA & C2C12 mouse myoblasts & Contractile tissue & SLA & Christensen et al. ${ }^{[83]}$ \\
\hline Silicone-modified copolymer & Human mesenchymal stem cells & Stem cell differentiation & Extrusion & Mohanty et al. ${ }^{[84]}$ \\
\hline $\begin{array}{l}\text { Hyaluronic acid-carboxymethylcel- } \\
\text { lulose }\end{array}$ & MC3T3 & Angiogenesis & Extrusion & Janarthanan et al. ${ }^{[85]}$ \\
\hline Collagen impregnated with MNPs & hMSC/MC3T3 & Osteogenesis & - & Jaiswal et al. $^{[88]}$ \\
\hline
\end{tabular}



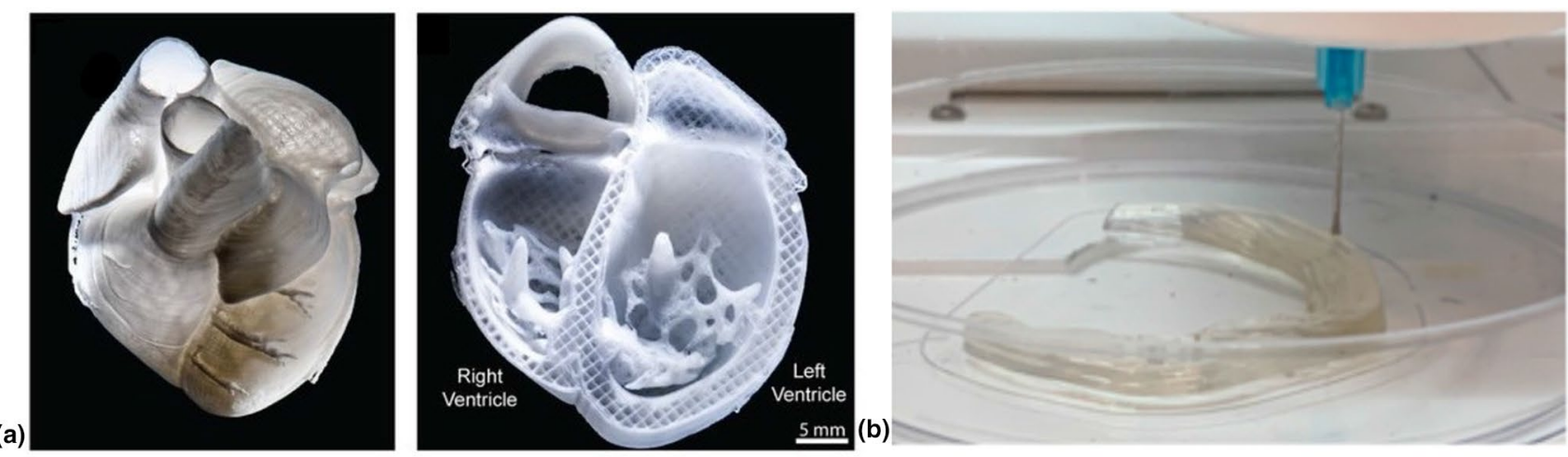

(c)
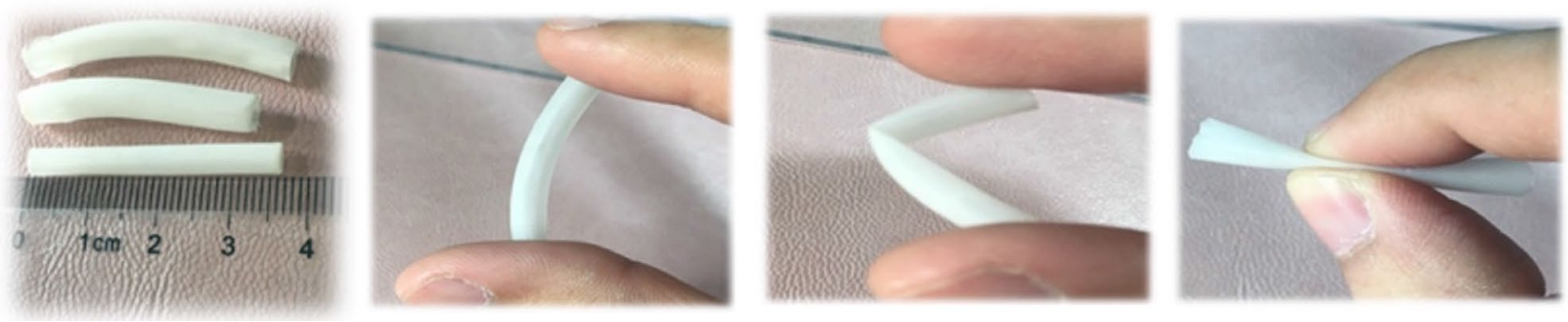

Figure 4. (a) 3D printed human heart replica using collagen-material. Left: fresh print, Right: cross-sectional view. ${ }^{\text {[90] }}$ (b) $3 \mathrm{D}$ printing an artificial meniscus ${ }^{[91]}$ Reprinted (adapted) with permission from ACS Appl. Polym. Mater. 2019, 1, 8, 2023-2032. Copyright (2019) American Chemical Society (c) Flexible 3D printed urethra tubes. ${ }^{[92]}$ Reprinted (adapted) with permission from ACS Appl. Bio Mater. 2020, 3, 4, 2007-2016. Copyright (2020) American Chemical Society.

Knee injuries are commonplace for athletes playing sports like soccer, football, and basketball. The repeated and abrupt directional change required to play these sports at a high-level breeds ample opportunity to damage the knee. The meniscus is a major ligament in the knee and can be described as having a wedge shape. While there are surgeries for repairing a torn meniscus, damage to this ligament can result in long-term chronic pain. Zhang et al. ${ }^{[91]}$ investigated the feasibility of 3D printing an artificial meniscus that can replace the ligament when torn or damaged. This artificial meniscus, termed as super strong, was 3D printed using cellulose nanocrystal, phenyl acrylate, and soft acrylamide. Figure $4 \mathrm{~b}$ shows $3 \mathrm{D}$ printing of an artificial meniscus. Producing a print with similar, if not better, properties than a real human meniscus is a promising result, but there are still challenges facing this type of application including effectively anchoring the meniscus to the bone and retention of material properties overtime. Nonetheless, these objectives are worth pursuing due to the societal and financial upside they represent.

The urethra is a tube-like structure that allows urine to exit the bladder. Congenital or acquired ailments of the urethra are a challenging and widespread issue for urologists. When the urethra becomes damaged or deformed, medical professionals experience great difficulty in repairing the damage and restoring its function. Procedures up to this point have largely relied on grafting tissue, which can work, but the graft can also be rejected. Xu et al. ${ }^{[92]}$ investigated the feasibility of using a three-component biocompatible polymer hydrogel in printing an artificial and biodegradable urethra tube. The hydrogel material is composed of triethyl citrate (TEC), polycaprolactone (PCL), and poly(lactic-co-glycolic acid) (PLGA) at a ratio of 6:30:70, respectively. As displayed in Fig. 4(c), the elastic behavior of the resulting print allows for shape restoration, and is thus, ideal for such application. If this material can be modified to the point where sustained acceptance and function are achieved in biological systems, then there is potential for a promising treatment to urethra ailments.

In addition, many extrusion-based printing techniques have been limited by their ability to fabricate high-resolution 3D-printed hydrogels, which currently still remains a challenge in the field of AM. Several approaches have been exploited to achieve high print resolution. For example, light-based 3D printing techniques such as DLP and SLA have been used, not only to print objects much faster, but with better spatial resolution, higher geometric complexity, and higher dimensional accuracy than FDM. ${ }^{[93-96]}$ Between the two techniques, DLP is more ideal in printing cell-laden hydrogels for tissue engineering applications requiring high resolution and cell viability as it employs visible light, while SLA irradiates the resin with UV light. However, the resolution on transparent resins can be reduced by undesired light penetration owing to the high transmissibility of the solution. Shin et al ${ }^{[95]}$ addressed this issue by introducing silk fibroin infused with melanin nanoparticles (SFM) to an otherwise transparent solution of poly(ethylene glycol)-tetraacrylate (PEG4A). The addition of SFM allows for the transparency of the solutions to be modulated such that the irradiation of the resin can be performed with higher precision. 
High print resolution using these photopolymerization-based approaches can also be achieved by inclusion of water-soluble photoblockers (e.g. chlorophyllin, tartrazine, etc.) capable of strongly absorbing light. ${ }^{[93]}$ Various dyes can also be added to further enhance the printing resolution. ${ }^{\left[{ }^{[4]}\right.}$ However, an increased curing time is required for the same layer thickness when higher dye concentrations are added. Likewise, two-photon polymerization (2PP) can be employed in fabricating up to 100-nm scale high-resolution printed structures for tissue engineering, drug delivery, biosensing, and other applications. ${ }^{[94]}$

Conversely, Hsieh et al. ${ }^{[96]}$ demonstrated the potential for high resolution, extrusion-based, 3D-printed hydrogels by printing various formulations of polyurethane-gelatin (PUgelatin) using nozzle diameters of 80,200 , and $320 \mu \mathrm{m}$. Cell proliferation was retained with marginal loss to cell viability in the hydrogel structures printed using $80 \mu \mathrm{m}$ nozzle relative to structures printed with 200 and $320 \mu \mathrm{m}$ diameters. The mechanical properties of the 3D printed constructs were further optimized by treatment with $\mathrm{Ca}^{2+}$ ions, followed by thermal curing. The introduction of $\mathrm{Ca}^{2+}$ induced a chelating effect within the PU-gelatin matrix and subsequently improved the structural integrity of the printed parts.

The point of the examples above is to show the need for a highly integrated approach in meeting the $3 \mathrm{D}$ printing process with the materials and function of the device (implant, transplant, orthesis, prosthesis). The ability of cells to proliferate and for the tissues to grow with vascularization means that they have to be incorporated early in the materials and the CAD or digital design of the device. The chemistry (addition of EGF, imprinting, theranostic function, etc.) can be part of the materials or added as a surface functionality. Thermomechanical properties whether as tissue scaffolds, orthopedic support, or prosthetic devices can take advantage of the right synthetic materials that have the tensile, compressive, and flexural modulus to support movement. The porosity of the materials and the CAD design of the printed geometry specifically the infill design or volume, can specify thermoplastic to elastomeric properties. Lastly, the hydrogel can also function as a surface coating material to promote adhesion, lubrication, and a host of biocompatible properties unique with a gel material. The need and design has to be a marriage with the biomedical function (clinically driven) and the materials/3D printing process (fabrication driven). There is an opportunity to integrate these relationships and parameters to address the unique needs of clinically-driven projects that will have an important biomedical engineering role in years to come.

\section{Future outlook}

Hydrogel 3D printing techniques have had significant technological improvements through the years, particularly in the development of materials and printing technologies. However, there are many important issues that still needs to be addressed in terms of taking these new developments in 3D printing chemistries, digitization, optimized printing methods, and clinical relevance into new heights. The outlook is outlined as follows:

\section{Materials properties}

During extrusion printing, the hydrogel material should have a suitable viscosity, extrudability and demonstrate shear-thinning behavior and should be easily extruded out of a nozzle. After printing, the hydrogel should exhibit high thixotropy and be able to maintain its shape (i.e. post-printing stability) and provide adequate mechanical structural support. Other properties that are worth investigating include interfacial adhesion, stackability, shape fidelity, bioactivity (e.g. promotion of cell viability, cell growth and cell proliferation), degradation properties (for in vitro culture/in vivo environments), ${ }^{[8]}$ biocompatibility, and biomimicry. ${ }^{[36]}$ In terms of materials properties, stimuli-responsiveness, shape memory function, and controlled resorbability, can also be defined by the digital design of the part (voxel resolution, infill design or volume, geometrical shape, etc.).

\section{Crosslinking methods}

The most commonly used crosslinking methods for hydrogels are photo and ionic crosslinking due to their relatively high crosslinking efficiency. New materials in conjunction with crosslinking methods are needed to be further developed. ${ }^{[97]}$ There is a need to differentiate pre- and post- printing chemistries that can further control the crosslinking density and hence, the thermomechanical properties. Non-covalent dynamic chemistry such as those found in vitrimers can be an interesting avenue towards hybrid mechanical properties as well as recylability. ${ }^{[97]}$

\section{Mixing/formulation of composites and nanocomposites}

Hydrogel composites are usually produced using various mixing processes, which could induce particle aggregation/agglomeration within the matrix. These agglomerations may lead to inhomogeneous printed composite materials/parts. Therefore, formulation of new methods ensuring uniform particle distribution is essential for real-world applications of 3D-printed hydrogel composites. ${ }^{[97]}$ Particles or fillers can include inorganic or organic materials with a minimum percolation threshold to achieve the property. Eventually, with the greater possibility of nanofillers (provided non-cytotoxicity is proven), nanostructuring of the particles and their dependence on the aspect ratio and interfacial chemistry will be depending on the optimized mixing or dispersion. This can enable new properties (thermally conducting, electrically conducting, sensing, catalytic, etc.) to be included in the original biomedical function of a hydrogel material.

\section{Mechanical anisotropy}

3D-printed parts can be intrinsically anisotropic due to the layer-by-layer printing fabrication. This behavior, not only 
directly affects the strength of pristine hydrogels, but also the continuity of particle reinforcements in hydrogel composites, whereby stronger parts are oriented parallel towards the directions parallel to the raster direction. ${ }^{[97]}$ If desired, anisotropy can be built on the fabricated part to control the strength with respect to specific axes of performance or repeated stress in usage. Also, the design can be part of the geometric and material integration early in order to build adequate/robust thermomechanical properties.

\section{Testing and simulation}

Due to the complex loading conditions of biomaterial implants in real-world applications, along with the aforementioned anisotropy and strong dependence of hydrogel mechanical properties on their internal structures, sample dimensions for mechanical testing should not be limited to conventional ISO/ ASTM testing standards (dog-bone, bar, etc.). ${ }^{[11,97]}$ Specific tests for hydrogel materials will also include stimuli responsiveness ( $\mathrm{pH}$, temperature, isoelectronic conditions, electrolyte balance, etc.). More importantly FDA approved properties and safety will be relevant for actual clinical application.

\section{Advances in printing technology and fabrication \\ Multi-material and co-axial printing}

Multi-material and co-axial printing allows printing of different materials with properties, thus creating parts with high structure/architectural complexities and diversity in properties and functionalities (i.e. increases heterogeneity). ${ }^{[37]}$ There is a possibility that this method can be extended to hydrogel and bioprinting, resulting in better geometry and distribution of material control.

\section{Combination of 3D printing and electrospinning}

Combining these two methods could make effective use of the intrinsic properties of each processing technique (i.e. the high porosity/low resolution of a 3D-printed part and tight/ultrafine intertwining of electrospun fibers). With these properties combined, pore sizes, surface topography, and mechanical properties may easily be tailored. ${ }^{[14,37]}$ Electrospinning can produce directionality of geometry along with anisotropy of thermomechanical properties. This configuration can be tailored to a particular implant device and hydrogel/collagen composition.

\section{Biofabrication}

Biofabrication here refers to the production of living and nonliving biological products from biomaterials, ECMs, molecules, and living cells. 3D printing is employed in the production of tissue-engineered devices and ECMs. ${ }^{[98]} 3 \mathrm{D}$ printing has been shown to enable the manufacture of structures having hierarchical tissue-like properties with customizable constructs. In addition, nanocomposite hydrogels developed from 3D printing exhibit anisotropic structures. Because they offer better control and ability to actively design the scaffold porosity and interconnectivity, solid free-form fabrication systems such as RP or fiber deposition technology are currently at the center of attention. These versatile systems can produce complex scaffolds with well-defined architectures and optimized pore interconnectivity. ${ }^{[65]}$ Biofabrication involves the use of bioinks, possessing high resolution printability with cytocompatibility. Although bioink formulation is a current issue, the inclusion of polymeric biomaterials, combined with thermoresponsive and photocrosslinkable biomaterial network, is expected to expand the quantity of available bioinks for biofabrication. ${ }^{[64,68,69,99]}$ $3 \mathrm{D}$ printing of supramolecular hydrogels may be employed to create scaffolds capable of stimulating the macroscopic alignment of cells and fabricate materials with anisotropic ionic and electronic conductivity. These materials can be made possible due to the synergistic capabilities of supramolecular self-assembly and $3 \mathrm{D}$ printing. ${ }^{[9,100]} \mathrm{A}$ consequence of this multi-material and multi-cellular strategy is the provision for new types of geometric orientation synergistic with the cell differentiation process.

\section{Microfluidics}

The following emerging technologies may also be considered: single-nozzle multi-material (SNMM) multimaterial multinozzle (MM3D), in-situ bioprinting, and combination of 3D printing and microfluidics. ${ }^{[37]}$ These combinations, in particular, can have promise on new types of materials delivery, but perhaps the ability to supply nutrients to specific cycles of cell growth and an alternative to vascularization.

\section{D printing}

The 4D bioprinting, using smart/stimuli-responsive materials, may also be used to create dynamic 3D-printed biological architectures capable of changing their sizes/shapes based on an applied stimulus. ${ }^{[10,14]}$ Materials with multi-sensitivities may also be an interesting research topic since the use of vitrimeric and shape-memory properties combined with the hydrogel materials function can enhance control. A synergistic design, with the changing shape or function, along with maturation of tissue growth, can be controlled with stimuli. Additionally, biomimetic 3D printing of tissues mimicking natural tissues may also be extensively explored which could also be related to $4 \mathrm{D}$ printing. ${ }^{[82]}$

Overall, 3D printed hydrogels are an ideal material for constructing biocompatible scaffolds for tissue engineering applications. The range of new printing methods, available materials, and potential applications make these materials a rich field for future research. While there are challenges facing these applications, the advancement of the field to this point is an encouraging sign that high progress and adaptation will be made in many research and clinical settings. 


\section{Summary}

This prospective initially presented the classification and applications of hydrogels, followed by the design considerations in the production of hydrogels (i.e. physical and biological parameters) and discussion on the material precursors (i.e. biopolymers, synthetic polymers and nanocomposites) for 3D-printing hydrogels. The combination of new methods of printing, multimaterial and multi-axes printing, along with $4 \mathrm{D}$ printing, will enable a more synergistic and hybrid approach in the application of hydrogel materials for tissue engineering. The future of this field demands collaborative and interdisciplinary efforts to overcome any fabrication obstacles. Lastly, future opportunities on the 3D printing of hydrogels in combination with new materials, new fabrication technologies, and clinical applications were highlighted.

\section{Acknowledgments}

This work is supported by the Department of Science and Technology_Philippine Council for Industry, Energy, and Emerging Technology Research and Development (DOST-PCIEERD) for JD, RM, FS, and AE and Bataan Peninsula State University for JD. The work by the students are also supported by the University of Tennessee and the Governor's Chair Fund. Work (or Part of this work) was conducted by ORNL's Center for Nanophase Materials Sciences by RCA, which is a US Department of Energy Office of Science User Facility.

\section{References}

1. A. Gregor, E. Filova et al., Designing of PLA scaffolds for bone tissue replacement fabricated by ordinary commercial 3D printer. J. Biol. Eng. 11(1), 31 (2017)

2. A.A.R. Lanza, R. Langer, J. Vacanti, Principles of Tissue Engineering (Academic Press, Cambridge, MA, 2011)

3. H.P.W.U. Meyer, T. Meyer, J. Handschel, Fundamentals of Tissue Engineering and Regenerative Medicine (Springer, Berlin, 2009)

4. Y.Z.X. Zhang, Tissue engineering applications of three-dimensional bioprinting. Cell Biochem. Biophys. 72, 777-782 (2015). https://doi.org/10. 1007/s12013-015-0531-x

5. D.X.B. Chen, Scaffold Design, in Extrusion Bioprinting Scaffolds Tissue Engineering Application 2019. (Springer, Berlin, 2019), pp. 15-30

6. C.A.M.P.A. Janmey, Cell mechanics: integrating cell responses to mechanical stimuli. Annu. Rev. Biomed. Eng. 9, 1-34 (2007). https://doi.org/10. 1146/annurev.bioeng. 9.060906 .151927

7. S.J. Hollister, Porous scaffold design for tissue engineering. Nat. Mater. $\mathbf{4}$, 518-524 (2005)

8. L.L.H. Li, C. Tan, Review of 3D printable hydrogels and constructs. Mater. Des. 159, 20-38 (2018)

9. J. Li, C. Wu, P.K. Chu, 3D printing of hydrogels: Rational design strategies and emerging biomedical applications. Mater. Sci. Eng. R 140, 100543 (2020)

10. K.Y.Z. Xia, S. Jin, Tissue and organ 3D bioprinting. SLAS Technol. 23, 301-314 (2018)

11. J.R.C. Dizon, A.H. Espera, Q. Chen, R.C. Advincula, Mechanical characterization of 3D-printed polymers. Addit. Manuf. (2018). https://doi.org/ 10.1016/j.addma.2017.12.002

12. A.C. De Leon, Q. Chen, N.B. Palaganas, J.O. Palaganas, J. Manapat, R.C. Advincula, High performance polymer nanocomposites for additive manufacturing applications. React. Funct. Polym. 103, 141-155 (2016). https://doi.org/10.1016/j.reactfunctpolym.2016.04.010

13. A.D. Valino, J.R.C. Dizon, A.H. Espera, Q. Chen, J. Messman, R.C. Advincula, Advances in 3D printing of thermoplastic polymer composites and nanocomposites. Prog. Polym. Sci. (2019). https://doi.org/10.1016/j.progp olymsci.2019.101162

14. L. D. Tijing, J. R. C. Dizon, I. Ibrahim, A. R. N. Nisay, H. K. Shon, and R. C. Advincula, "3D printing for membrane separation, desalination and water treatment," Appl. Mater. Today, vol. 18, 2020, doi: https://doi.org/10.1016/j. apmt.2019.100486.

15. A.H. Espera, J.R.C. Dizon, Q. Chen, R.C. Advincula, 3D-printing and advanced manufacturing for electronics. Prog. Addit. Manuf. (2019). https://doi.org/10.1007/s40964-019-00077-7

16. R.N.R.C. Advincula, J.R.C. Dizon, Q. Chen, I. Niu, J. Chung, L. Kilpatrick, Additive manufacturing for COVID-19: devices, materials, prospects, and challenges. MRS Commun. 10(3), 413-427 (2020)

17. D.C. Siacor, Q. Chen, J.Y. Zhao, L. Han, A.D. Valino, E.B. Taboada, E.B. Caldona, On the Additive Manufacturing (3D Printing) of viscoelastic materials and flow behavior: From composites to food manufacturing. Addit. Manuf. 45, 102043 (2021). https://doi.org/10.1016/j.addma. 2021.102043

18. H.S. Agueda, Q. Chen, R.D. Maalihan, J. Ren, Í.G. da Silva, N.P. Dugos, E.B. Caldona, 3D printing of biomedically relevant polymer materials and biocompatibility. MRS Commun. (2021). https://doi.org/10.1557/ s43579-021-00038-8

19. C.K. Chua, K.F. Leong, 3D Printing and Additive Manufacturing: Principles and Applications, 5th edn. (World Scientific, Singapore, 2017)

20. J.J. Ballyns, J.P. Gleghorn, V. Niebrzydowski, J.J. Rawlinson, H.G. Potter, S.A. Maher, T.M. Wright, Image-guided tissue engineering of anatomically shaped implants via MRI and micro-CT using injection molding. Tissue Eng. A 14(7), 1195-1202 (2008)

21. H.N. Chia, B.B. Wu, Recent advances in $3 \mathrm{D}$ printing of biomaterials. J Biol Eng 9(1), 4 (2015). https://doi.org/10.1186/S13036-015-0001-4

22. H.H. Seyednejad, D. Gawlitta, R.V. Kuiper, A. DeBruin, C.F. van Nostrum, T. Vermonden, W.J.A. Dhert, In vivo biocompatibility and biodegradation of 3D-printed porous scaffolds based on a hydroxyl-functionalized poly(epsiloncaprolactone). Biomaterials 33(17), 4309-4318 (2012). https:// doi.org/10.1016/j.biomaterials.2012.03.002

23. G.H. Wu, S.H. Hsu, Review: polymeric-Based 3D printing for tissue engineering. J Med. Bioeng. 35(3), 285-292 (2015). https://doi.org/10.1007/ s40846-015-0038-3

24. S. Utech, A.R. Boccaccini, A review of hydrogel-based composites for biomedical applications: enhancement of hydrogel properties by addition of rigid inorganic fillers. J Mater Sci 51(1), 271-310 (2016). https://doi.org/ $10.1007 / \mathrm{s} 10853-015-9382-5$

25. A.K. Gaharwar et al., Nanocomposite hydrogels for biomedical applications. Biotechnol. Bioeng. 111(3), 441-453 (2014). https://doi.org/10.1002/bit.25160

26. G.R. Singhal, A review: tailor-made hydrogel structures (classifications and synthesis parameters). Polym. Plast. Technol. Eng. 55, 54-70 (2016). https://doi.org/10.1080/03602559.2015.1050520

27. J.-H. Lee, H.-W. Kim, Emerging properties of hydrogels in tissue engineering. J. Tissue Eng. (2018). https://doi.org/10.1177/2041731418768285

28. C.D. Spicer, Hydrogel scaffolds for tissue engineering: the importance of polymer choice. Polym. Chem. 11, 184-219 (2020)

29. E.M. Ahmed, Hydrogel: preparation, characterization, and applications: a review. J. Adv. Res. 6(2), 105-121 (2015). https://doi.org/10.1016/j.jare. 2013.07.006

30. D.X.B. Chen, Biomaterials for bioprinting, in Extrusion bioprinting scaffolds tissue engineering application. (Springer, Dordrecht, 2019), pp. 33-48

31. A.S.-N.A. Vedadghavami, F. Minooei, M.H. Mohammadi, S. Khetani, A.R. Kolahchi, S. Mashayekhan, Manufacturing of hydrogel biomaterials with controlled mechanical properties for tissue engineering applications. Acta Biomater. 62, 42-63 (2017). https://doi.org/10.1016/j.actbio.2017.07.028

32. R.E.M.J. Zhu, Design properties of hydrogel tissue-engineering scaffolds. Expert Rev. Med. Devices 8(5), 607-626 (2011). https://doi.org/10.1586/ erd. 11.27

33. G.G.W.J.H.Y. Chung, S. Naficy, Z. Yue, R. Kapsa, A. Quigley, S.E. Moulton, Bio-ink properties and printability for extrusion printing living cells. Biomater. Sci. 1, 763-773 (2013). https://doi.org/10.1039/c3bm00012e 
34. X.C.F. You, B.F. Eames, Application of extrusion-based hydrogel bioprinting for cartilage tissue engineering. Int. J. Mol. Sci. 18, 8-14 (2017). https:// doi.org/10.3390/ijms18071597

35. H. Tetsuka, S.R. Shin, Materials and technical innovations in 3D printing in biomedical applications. J. Mater. Chem. B 8(15), 2930 (2020)

36. A.A.S.V. Murphy, 3D bioprinting of tissues and organs. Nat. Biotechnol 32, 773-785 (2014). https://doi.org/10.1038/nbt.2958

37. M.M. Askari, M.A. Naniza, M. Kouhic, A. Saberid, A. Zolfagharian, M. Bodaghia, Recent progress in extrusion 3D bioprinting of hydrogel biomaterials for tissue regeneration: a comprehensive review with a focus on advanced fabrication techniques". Biomater. Sci. 9, 535-573 (2021). https://doi.org/10.1039/D0BM00973C

38. C.B. Highley, C.B. Rodell, Direct 3D printing of shear-thinning hydrogels into self-healing hydrogels. Adv. Mater 27(34), 5075-5079 (2015). https:// doi.org/10.1002/adma.201501234

39. P.C.S. Naahidi, M. Jafari, M. Logan, Y. Wang, Y. Yuan, H. Bae, B. Dixon, Biocompatibility of hydrogel-based scaffolds for tissue engineering applications. Biotechnol. Adv. 35(5), 530-544 (2017). https://doi.org/10.1016/j. biotechadv.2017.05.006

40. T.S.M.M. Khansari, L.V. Sorokina, P. Mukherjee, F. Mukhtar, M.R. Shirdar, M. Shahidi, Classification of hydrogels based on their source: A review and application in stem cell regulation. J0M 69, 1340-1347 (2017). https:// doi.org/10.1007/s11837-017-2412-9

41. M.H.Y.I.M. El-Sherbiny, Hydrogel scaffolds for tissue engineering: Progress and challenges. Glob. Cardiol. Sci. Pract. 2013(3), 316-334 (2013). https:// doi.org/10.5339/gcsp.2013.38

42. Q.L.H. Yan, X. Chen, M. Feng, Z. Shi, D. Zhang, Layer-by-layer assembly of $3 \mathrm{D}$ alginate-chitosan-gelatin composite scaffold incorporating bacterial cellulose nanocrystals for bone tissue engineering. Mater. Lett. 209, 492-496 (2017). https://doi.org/10.1016/j.matlet.2017.08.093

43. Z.J.J. He, R. Chen, Y. Lu, L. Zhan, Y. Liu, D. Li, Fabrication of circular microfluidic network in enzymatically-crosslinked gelatin hydrogel. Mater. Sci. Eng. C 59, 53-60 (2016). https://doi.org/10.1016/j.msec.2015.09.104

44. A.R. Brunelle, C.B. Horner, K. Low, G. Ico, Electrospun thermosensitive hydrogel scaffold for enhanced chondrogenesis of human mesenchymal stem cells. Acta Biomater. 66, 166-176 (2018). https://doi.org/10.1016/j. actbio.2017.11.020

45. P.K. Nguyen, W. Gao, D. Patel, Z. Siddiqui, S. Weiner, E. Shimizu, B. Sarkar, Self-assembly of a dentinogenic peptide hydrogel. ACS Omega 3(6), 59805987 (2018). https://doi.org/10.1021/acsomega.8b00347

46. O.G.N.M. Ergul, S. Unal, I. Kartal, C. Kalkandelen, N. Ekren, O. Kilic, L. Chi-Chang, 3D printing of chitosan/poly(vinyl alcohol) hydrogel containing synthesized hydroxyapatite scaffolds for hard-tissue engineering. Polym. Test. 79, 106006 (2019). https://doi.org/10.1016/j.polymertesting.2019. 106006

47. L. Anja, M. Michael, B. Sophie, P. Birgit, B. Hagen, S. Michaela, W. Claudia, S. Frank, Additive manufacturing of collagen scaffolds by three-dimensional plotting of highly viscous dispersions. Biofabrication 8(1), 015015 (2016)

48. J.S.T. Jang, H. Jung, H. Pan, W. Han, S. Chen, 3D printing of hydrogel composite systems: recent advances in technology for tissue engineering. Int. J. Bioprinting 4(1), 126 (2018)

49. J.W.W. Hu, Z. Wang, Y. Xiao, S. Zhang, Advances in crosslinking strategies of biomedical hydrogels. Biomater. Sci. 7, 843-855 (2019). https://doi.org/ 10.1039/C8BM01246F

50. D.S.L.C.T. Huynh, M.K. Nguyen, Injectable block copolymer hydrogels: achievements and future challenges for biomedical applications. Macromolecules 44(17), 6629-6636 (2011). https://doi.org/10.1021/ma201 $261 \mathrm{~m}$

51. F.A.P. Matricardi, C. Di Meo, T. Coviello, W.E. Hennink, Interpenetrating polymer networks polysaccharide hydrogels for drug delivery and tissue engineering. Adv. Drug Deliv. Rev. 65(9), 1172-1187 (2013). https://doi. org/10.1016/j.addr.2013.04.002

52. S.S.S. Goenka, V. Sant, Graphene-based nanomaterials for drug delivery and tissue engineering. J. Control. Release 173, 75-88 (2014). https://doi. org/10.1016/j.jconrel.2013.10.017

53. M.R.H.R. Eivazzadeh-Keihan, K.K. Chenab, R. Taheri-Ledari, J. Mosafer, S.M. Hashemi, A. Mokhtarzadeh, A. Maleki, Recent advances in the application of mesoporous silica-based nanomaterials for bone tissue engineering. Mater. Sci. Eng. C 107, 110267 (2020). https://doi.org/10. 1016/j.msec.2019.110267

54. B.Z.J. Saroia, W. Yanen, Q. Wei, K. Zhang, T. Lu, A review on biocompatibility nature of hydrogels with $3 \mathrm{D}$ printing techniques, tissue engineering application and its future prospective. Bio-des. Manuf. 1, 265-279 (2018). https://doi.org/10.1007/s42242-018-0029-7

55. D. Williams, Revisiting the definition of biocompatibility. Med. Dev. Technol. 14(8), 10 (2003)

56. S.H.T.D.W. Hutmacher, J.C. Goh, An introduction to biodegradable materials for tissue engineering applications. Ann. Acad. Med. Singap. 30(2), 183-191 (2001)

57. D.J.M.J.L. Drury, Hydrogels for tissue engineering: scaffold design variables and applications. Biomaterials 24, 4337-4351 (2003)

58. J. Rouwkema, N.C. Rivron, C.A. van Blitterswijk, Vascularization in tissue engineering. Trends Biotechnol. 26(8), 434-441 (2008). https://doi.org/ 10.1016/j.tibtech.2008.04.009

59. H.H.H.D. Druecke, S. Langer, E. Lamme, J. Pieper, M. Ugarkovic, H.U. Steinau, Neovascularization of poly(ether ester) block-copolymer scaffolds in vivo: long-term investigations using intravital fluorescent microscopy. Biomed. Mater. Res. A 68, 10-18 (2004)

60. C.K.C.S. Yang, K.F. Leong, Z. Du, The design of scaffolds for use in tissue engineering. Part I. Traditional factors. Tissue Eng. 7, 679-689 (2001)

61. X. Bai, M. Gao, S. Syed, J. Zhuang, X. Xu, Bioactive hydrogels for bone regeneration. Bioact. Mater. 3(4), 401-417 (2018). https://doi.org/10. 1016/j.bioactmat.2018.05.006

62. Y. Kim, H. Ko, K. Kwon, Extracellular matrix revisited: roles in tissue engineering. Int Neurourol J 20, S23-29 (2016). https://doi.org/10.5213/ inj.1632600.318

63. H.T.T.F. Akther, P. Little, Z. Li, N.T. Nguyen, Hydrogels as artificial matrices for cell seeding in microfluidic devices. RSC Adv 10, 43682-43703 (2020). https://doi.org/10.1039/D0RA08566A

64. S.D.T.S. Mantha, S. Pillai, P. Khayambashi, A. Upadhyay, Y. Zhang, 0. Tao, H.M. Pham, Smart hydrogels in tissue engineering and regenerative medicine. Materials 12(20), 3323 (2019). https://doi.org/10.3390/ ma12203323

65. P.D.T. Billiet, M. Vandenhaute, J. Schelfhout, S. Van Vlierberghe, A review of trends and limitations in hydrogel-rapid prototyping for tissue engineering. Biomaterials 33(26), 6020-6041 (2012). https://doi.org/10.1016/j.bioma terials.2012.04.050

66. A.K.J. Leijten, J. Seo, K. Yue, G. Trujillo-de Santiago, A. Tamayol, G.U. RuizEsparza, S.R. Shin, R. Sharifi, I. Noshadi, M.M. Alvarez, Y.S. Zhang, Spatially and temporally controlled hydrogels for tissue engineering. Mater. Sci. Eng. R 119, 1-35 (2017). https://doi.org/10.1016/j.mser.2017.07.001

67. E. Jabbari, Challenges for natural hydrogels in tissue engineering. Gels 5(2), 30 (2019). https://doi.org/10.3390/gels5020030

68. R.P.H. Zhao, M. Liu, Y. Zhang, J. Yin, Nanocomposite hydrogels for tissue engineering applications. Nanoscale 12(28), 14976-14995 (2020). https:// doi.org/10.1039/DONR03785K

69. S.G.K.S. Stratton, N.B. Shelke, K. Hoshino, S. Rudraiah, Bioactive polymeric scaffolds for tissue engineering. Bioact. Mater. 1, 93-108 (2016). https:// doi.org/10.1016/j.bioactmat.2016.11.001

70. F.D.N. Annabi, J.W. Nichol, X. Zhong, C. Ji, S. Koshy, A. Khademhosseini, Controlling the porosity and microarchitecture of hydrogels for tissue engineering. Tissue Eng. Part B 16(4), 371-383 (2010). https://doi.org/ 10.1089/ten.ten.2009.0639

71. H.F.C. Wu, A. Liu, S. Chen, X. Zhang, L. Chen, Y. Zhu, Z. Xiao, J. Sun, H. Luo, Cell-laden electroconductive hydrogel simulating nerve matrix to deliver electrical cues and promote neurogenesis. ACS Appl Mater Interfaces 11, 22512-22163 (2019). https://doi.org/10.1021/acsami.9b05520

72. F.B.E.K. Roshanbinfar, L. Vogt, B. Greber, S. Diecke, A.R. Boccaccini, T. Scheibel, Electroconductive biohybrid hydrogel for enhanced maturation and beating properties of engineered cardiac tissues. Adv. Funct. Mater. 28(42), 1803951 (2018). https://doi.org/10.1002/adfm.201803951

73. M.N.A. Navaei, N. Moore, R.T. Sullivan, D. Truong, R.Q. Migrino, Electrically conductive hydrogel-based micro-topographies for the development of organized cardiac tissues. RSC Adv. 7(6), 3302-3312 (2017). https://doi. org/10.1039/C6RA26279A 
74. S.A.B.Z.J. Rogers, M.P. Zeevi, R. Koppes, Electroconductive hydrogels for tissue engineering: current status and future perspectives. Bioelectricity 2(3), 279-292 (2020). https://doi.org/10.1089/bioe.2020.0025

75. J.-K.Y.L. Dong, S.-J. Wang, X.-R. Zhao, Y.-F. Zhu, 3D-printed poly ( $\varepsilon$-caprolactone) scaffold integrated with cell-laden chitosan hydrogels for bone tissue engineering. Sci. Rep. 7(1), 1 (2017)

76. C.L.M. Beldjilali-Labro, A. Garcia Garcia, F. Farhat, F. Bedoui, J.-F. Grosset, $M$. Dufresne, Biomaterials in tendon and skeletal muscle tissue engineering: current trends and challenges. Materials (Basel) 11(7), 1116 (2018)

77. M.P.C.A.D. Nocera, R. Comín, N.A. Salvatierra, Development of 3D printed fibrillar collagen scaffold for tissue engineering. Biomed. Microdevices 20(2), 1 (2018)

78. A.F.B.V. Martin, I.A. Ribeiro, M.M. Alves, L. Gonçalves, R.A. Claudio, L. Grenho, M.H. Fernandes, P. Gomes, C.F. Santos, Engineering a multifunctional 3D-printed PLA-collagen-minocycline-nanoHydroxyapatite scaffold with combined antimicrobial and osteogenic effects for bone regeneration. Mater. Sci. Eng. C 101, 15-26 (2019)

79. A.H.R. Murphy, D.P. Walsh, C.A. Hamilton, S.-A. Cryan, M. in het Panhuis, Degradable 3D-printed hydrogels based on star-shaped copolypeptides. Biomacromol 19(7), 2691 (2018)

80. S.C.P. Wang, D. Berry, A. Moran, F. He, T. Tam, L. Chen, Controlled growth factor release in 3D-printed hydrogels. Adv. Heal. Mater. 9(15), 1900977 (2020)

81. X.C.Q. Liu, Q. Li, S. Xu, Q. Zheng, Preparation and properties of 3D printed alginate-chitosan polyion complex hydrogels for tissue engineering. Polymers (Basel) 10(6), 664 (2018)

82. J.K. Tessmar, A.M. Göpferich, Customized PEG-derived copolymers for tissue-engineering applications. Macromol. Biosci. 7(1), 23 (2007)

83. K. Christensen, C. von Halling Laier, A. Kiziltay, S. Wilson, 3D printed hydrogel multiassay platforms for robust generation of engineered contractile tissues. Biomacromol 21(2), 356 (2019)

84. J.E.S. Mohanty, M. Alm, M. Hemmingsen, A. Dolatshahi-Pirouz, J. Trifol, P. Thomsen, M. Dufva, A. Wolff, 3D printed silicone-hydrogel scaffold with enhanced physicochemical properties. Biomacromol 17(4), 131 (2016)

85. G. Janarthanan, H.S. Shin, I.-G. Kim, P. Ji, E.-J. Chung, C. Lee, Selfcrosslinking hyaluronic acid-carboxymethylcellulose hydrogel enhances multilayered 3D-printed construct shape integrity and mechanical stability for soft tissue engineering. Biofabrication 12(4), 045026 (2020)

86. W.L.X. Zhai, Y. Ma, C. Hou, F. Gao, Y. Zhang, C. Ruan, H. Pan, W.W. Lu, 3D-printed high strength bioactive supramolecular polymer/clay nanocomposite hydrogel scaffold for bone regeneration. ACS Biomater. Sci. Eng. 3(6), 1109 (2017)

87. M. Håkansson, I.C. Henriksson, C. de la Peña Vázquez, V. Kuzmenko, K. Markstedt, P. Enoksson, Solidification of 3D printed nanofibril hydrogels into functional 3D cellulose structures. Adv. Mater. Technol. 1(7), 1600096 (2016)
88. M.K. Jaiswal, J.R. Xavier, J.K. Carrow, P. Desai, D. Alge, Mechanically stiff nanocomposite hydrogels at ultralow nanoparticle content. ACS Nano 10(1), 246 (2016)

89. P. Ramiah, L.C. du Toit, Y.E. Choonara, V. Pillay, Hydrogel-based bioinks for 3D bioprinting in tissue regeneration. Front. Mater. (2020). https://doi.org/ 10.3389/fmats.2020.00076

90. A.F.A. Lee, A. Hudson, D. Shiwarski, J. Tashman, T. Hinton, S. Yerneni, J. Bliley, P. Campbell, 3D bioprinting of collagen to rebuild components of the human heart. Science 365(6452), 482 (2019)

91. S.W.Z. Zhang, R. Liu, H. Zepeda, L. Zeng, J. Qiu, 3D printing super strong hydrogel for artificial meniscus. ACS Appl. Polym. Mater. 1(8), 2023 (2019)

92. J.Y.Y. Xu, Q. Meng, X. Jin, F. Liu, Biodegradable scaffolds for urethra tissue engineering based on 3D printing. ACS Appl. Bio Mater. 3(4), 2007 (2020)

93. A.D. Benjamin, R. Abbasi, M. Owens, R.J. Olsen, D.J. Walsh, T.B. LeFevre, Light-based 3D printing of hydrogels with high-resolution channels. Biomed. Phys. Eng. Express 5(2), 025035 (2019)

94. Q.G. Biao Zhang, S. Li, H. Hingorani, A. Serjouei, L. Larush, A.A. Pawar, W.H. Goh, A.H. Sakhaei, M. Hashimoto, K. Kowsari, S. Magdassi, Highly stretchable hydrogels for UV curing based high-resolution multimaterial 3D printing. J. Mater. Chem. B 6(20), 3246 (2018)

95. J.H.S. Shin, H. Kwak, Melanin nanoparticle-incorporated silk fibroin hydrogels for the enhancement of printing resolution in 3D-projection stereolithography of poly (ethylene glycol)-tetraacrylate bio-ink. ACS Appl. Mater. Interfaces 10(28), 23573 (2018)

96. C.-T. Hsieh, S. Hsu, Double-network polyurethane-gelatin hydrogel with tunable modulus for high-resolution $3 \mathrm{D}$ bioprinting. ACS Appl. Mater. Interfaces 11(36), 32746 (2019)

97. J.S.T.S. Jang, H.D. Jung, H.M. Pan, W.T. Han, S. Chen, 3D printing of hydrogel composite systems: recent advances in technology for tissue engineering. Int. J. Bioprint. 4(1), 126 (2018). https://doi.org/10.18063/IJB.v4i1.126

98. V.Y.S. Patra, A review of $3 \mathrm{D}$ printing techniques and the future in biofabrication of bioprinted tissue. Cell Biochem. Biophys. 74(2), 93-98 (2016). https://doi.org/10.1007/s12013-016-0730-0

99. M.E.G.M. Gomez-Florit, A. Pardo, R.M.A. Domingues, A.L. Graca, P.S. Babo, R.L. Reis, Natural-based hydrogels for tissue engineering applications. Molecules 25, 5858 (2020). https://doi.org/10.3390/molecules25245858

100. W.J.N.A. Sather, H. Sai, I.R. Sasselli, K. Sato, R.R.K.C.V. Synatschke, R.T. Zambrotta, J.F. Edelbrock, S.I.S.J.O. Hardin, J.D. Berrigan, M.F. Durstock, P. Mirau, 3D printing of supramolecular polymer hydrogels with hierarchical structure. Small 17, 2005743 (2021). https://doi.org/10.1002/smll.20200 5743 